\title{
BIM FOR CULTURAL HERITAGE
}

\author{
Matteo Del Giudice ${ }^{1}$, Anna Osello ${ }^{1}$ \\ ${ }^{1}$ Department of Structural, Geotechnical and Building Engineering (DISEG) - Politecnico di Torino, C.so Duca degli \\ Abruzzi 24, 10100, Torino, Italy - matteo.delgiudice@polito.it, anna.osello@polito.it
}

KEY WORDS: BIM, Point Clouds, survey, interoperability

\begin{abstract}
:
When you think about the Architecture, Engineering and Construction (AEC) Industry people tend to refers to new buildings, but nowadays the recovery of existing ones is increasingly the subject of the research. The current historical context raises this issue at the center of numerous thought due both to economic and environmental conditions. So, the need to refurbish the cultural heritage is becoming more important than the construction of new buildings. Modern technologies allow professionals to do this to turn the buildings into structures capable to meet the users' confort with a considerable energy saving. Italy is trying to make a change to the construction industry through the national InnovANCE project, which aims to develop the first national database able to share information among professionals through the help of Building Information Modeling (BIM). In this way the subject involved in a construction process can update their way of working, with a consequent time and cost saving. This paper aims to present the way in which the InnovANCE project can be considered as the key for Italy to change the way to conceive the building industry, using a case study such as the old thermal power of Politecnico di Torino, starting from the survey step. The methodology followed to obtain the 3D model will be described, starting from the data of a topographic and a laser scanner survey and from an archival documents research.
\end{abstract}

\section{INTRODUCTION}

The world environmental and economic conditions, focusing on Europe and specially on Italy, require a change of the development strategies of the society starting from the built environment in which people live.

Usually the AEC Industry is focusing on new buildings without paying attention to the cultural building heritage. So, the need to refurbish existing buildings, transforming them into smart buildings, is an essential research field in Italy in the last years. We know that the term 'smart buildings' describes a suite of technologies used to make the design, construction and operation of buildings more efficient, applicable to both existing and new-build properties. These might include Building Management Systems (BMS) that run lighting, heating and cooling systems according to occupants' needs or software that switches off all PCs and monitors after everyone has gone home. Of course, BMS data can be used to identify additional opportunities for efficiency improvements.

Adding to this, we can consider a smart building, as intelligent building, not only for a number of technologies that it contains, but also analyzing the method used to make the building, starting from the design phase arriving to management phase. This idea goes well with Building Information Modeling (BIM). Recently smart buildings has received much attention as well as BIM and interoperability as independent fields. To link these topics is an essential research target to help designers and stakeholders to run processes more efficiently. As a matter of fact, to work on a smart building requires the use of ICT to optimize design, construction and management. Nowadays, several technologies such as sensors for remote monitoring and control, building equipment, management software, etc. are available in the market. As BIM provides an enormous amount of information in its database and theoretically it is able to work with all kind of data sources using interoperability, it is essential to define standards for both data contents and format exchange. In this way, a possibility to align research activity with Horizon 2020 is the investigation of energy saving using ICT, and BIM and interoperability can play a key role to transform existing buildings into smart buildings. This idea was reflected in the InnovANCE project which aims to develop the first national database for the AEC Industry. This archive will be able to involve all professionals in the construction process, deleting misunderstandings that generate inefficiencies, optimizing each step of the process in term of energy saving and price performance. The use of this database is strictly related to the use of BIM which gives the possibility to manage all the construction process, reducing errors and costs. Although this project has many positive aspects there are still some difficulties related to the full creation of this idea.

First of all there is the need to change the way to concept the project because today professionals are used to work with CAD tools, secondly the BIM process is used for new building rather than for existing building. This is related to the fact that existing buildings have many constrains to respect, considering the will to preserve the correspondence between the parametric model and the existing structure. Adding to this the interoperability process is not error free.

To overcome these problems would be enough to train professionals according to this new methodology applied to existing building, using the InnovANCE database correctly. To solve problems related to sharing of data it is necessary to use the correct standard exchange formats and a correct modeling. Other states such as the UK have proceeded with the development of national database of BIM objects, called National BIM Library (NBL), to streamline the building process, setting a common approach to quality standards throughout the AEC industry. The NBL has the task to promote greater consistency of information improving the collaboration 
between professionals. At the end of the InnovANCE project (July 2015) all of this will be possible in Italy too.

The purpose of this database is to improve the sharing of information between different professionals within a project, solving the interferences between them, avoiding the creation of mistakes, decreasing costs, obtaining good results.

Relating to existing buildings, the design steps have been simulated with the BIM methodology in a traditional professional studio used to work CAD tools. Starting from an international bibliographic research and a more detailed plan for the state of the art of BIM in the most advanced countries, all the design steps have been set in the construction project to facilitate the transition from CAD to BIM.

At the beginning of the InnovANCE project, a case study has been selected: the old thermal power plant of the Politecnico di Torino. This is a building square shape built in the mid-50s: the structure will change its native function because four large classrooms reserved for teaching will be made inside it.

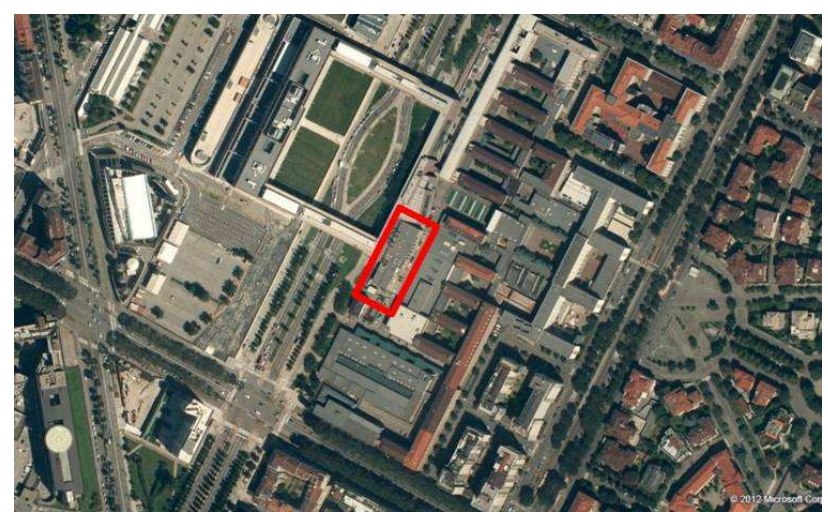

Figure 1. Location of the old thermal power(image extracted from Google Maps)

At present the database is continuously implemented and improved with a lot of tests using different case studies to find the easy way that will be followed by the professionals which want to use the InnovANCE database.

\section{METHODOLOGY}

During the first phase of the InnovANCE project time was spent to set properly the methodology that will be followed in the future of the project, beginning to develop the parametric model. More tests are needed to test the 3D model in specific applications, deepening the interoperability issue, arriving to define guidelines for the correct use of BIM for the refurbishment of the existing buildings. Existing building have a number of major difficulties than new buildings, but the use of new technologies mentioned above gives the opportunity for professionals to manage data, querying the 3 Dmodel through a iterative and interactive steps that characterize the BIM process. The case study analyzed consists of the refurbishment of the former thermal power plant of Politecnico di Torino. This choice joins the current need in which the issue of the recovery of buildings is also a priority in government policies. Adding to this, there is the need by the university to increase the numbers of rooms that can be used for educational purposes.

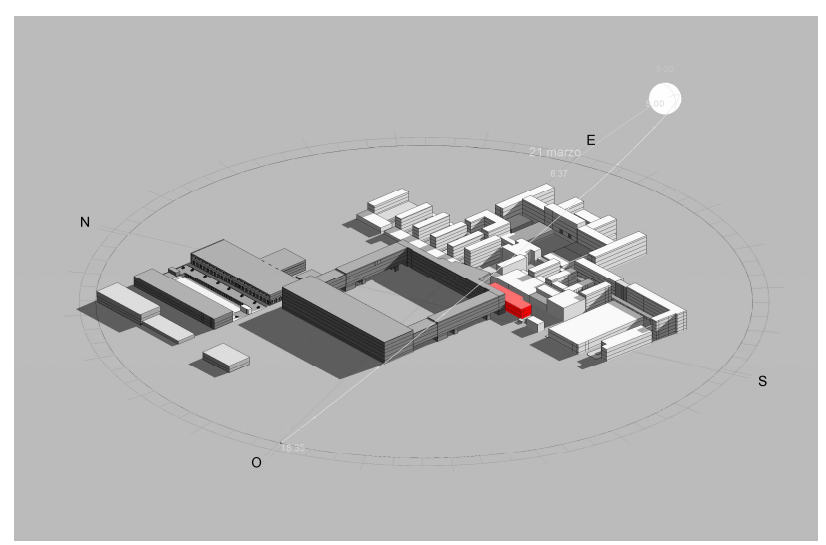

Figure 2. 3D Massing Model of Politecnico di Torino for shading study

The building is located adjacent to the newly constructed building that forms a part of the Doubled of the university. It has the east-west orientation that can be exploited in the design phase for natural building. The structure will change its native function because four large classrooms reserved for teaching will be made inside it.

Based on what we just said before the research has been organized and developed considering three essential elements:

- The working methodology based on the data exchange following the interoperable way and the problems' solution related to it;

- The data hierarchy during the different phases of the design and the role of professionals in the filling's data steps in the 3D model;

- The existing rules and the need to update them.

So, in order to organize the work to be performed, possible areas concerning the project management, in which it is necessary share information to develop the full BIM, have been identified.

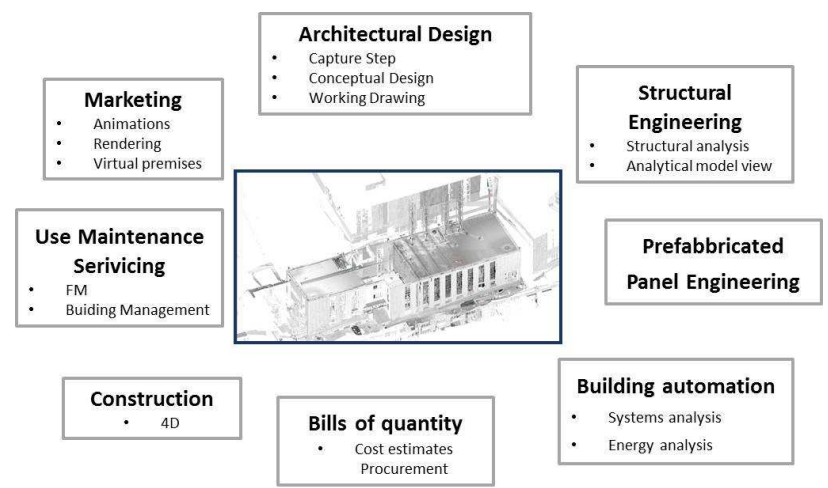

Figure 2. Organization of the building process As seen from the above figure, eight areas have been identified; at present we have focused on the Architectural design step. First of all, due the importance of the context analysis, a research based on the archival documents (by our team) and a geometric survey (by an external team) have been done. The geometric data capture needed for the developing of the parametric model in Autodesk Revit. This step took place in two parallel and complementary ways: the first way was characterized by a topographic survey using a total station for the measurement of the essential elements of the external facades, while the second by the use of laser scanner for the external and internal part of the building. 


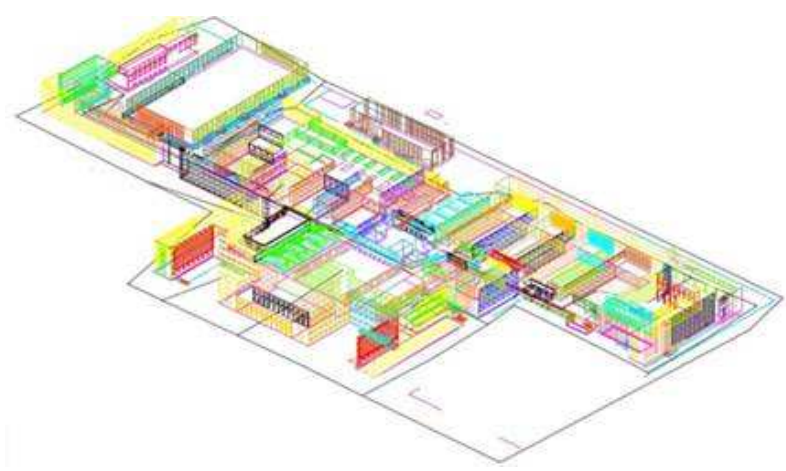

Figure 3. Survey of the Main Campus realized using a total station. (realized by Eraldo Mondino and Daniele Zaccaria)

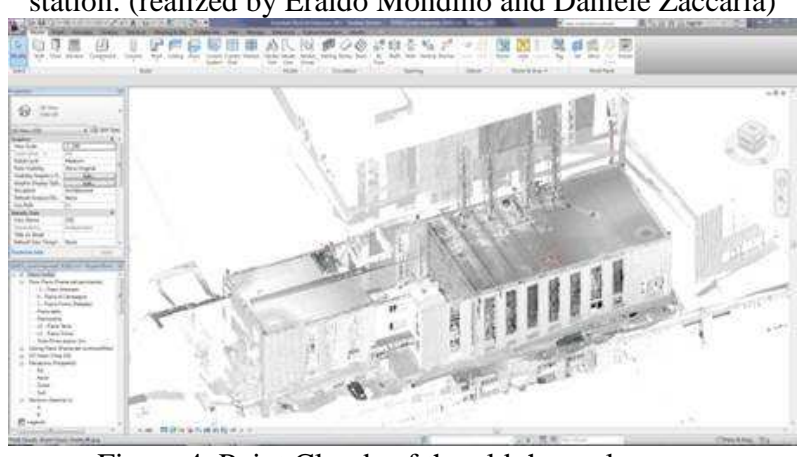

Figure 4. Point Clouds of the old thermal power.

(realized by A.Lingua charge from the construction and logistic area of the Politecnico di Torino)

The reason of these two way is due to the fact that it was necessary to check data coming from different sources, using the BIM process. The use of laser scanner to obtain the point clouds was fundamental for the correct location and orientation of the model: this should facilitate the subsequent lighting simulations. Adding to this, the point clouds of the internal part of the building have helped us to observe inconsistencies between the design documents and the existing building.

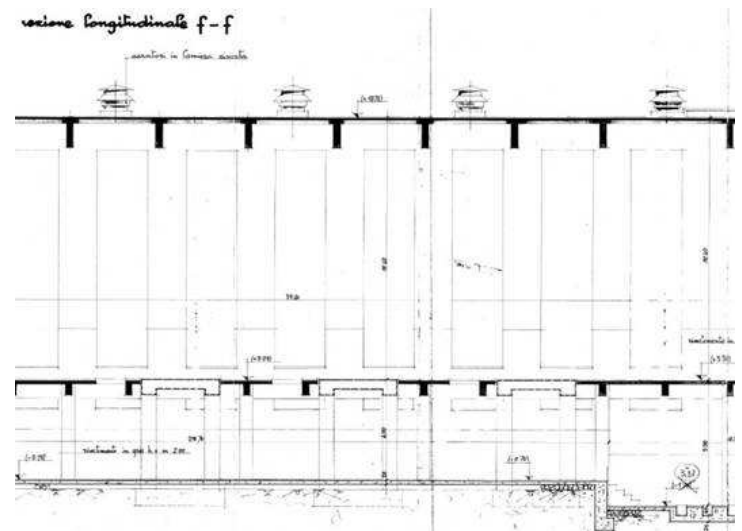

Figure 5. Longitudinal section of the old thermal power, coming from archival documents.

At the same time an historical research was conducted to find the original project which are essential to check the coherence with the building constructed.

This comparison showed some differences like for example the beam system of the principal floor modified to put the boilers inside of the structure. Following this procedure we obtained some information useful for the concrete reinforcement. In order to simulate a real situation and to optimize the information sharing between different professionals involved in a project worksets has been created, developing the information exchange idea. Worksharing is a design method that allows multiple team member to work on the same project model at the same time.

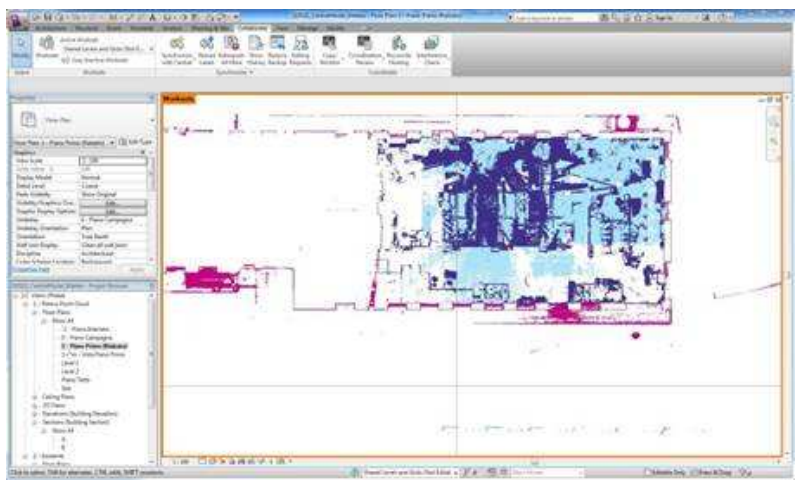

Figure 6. Image of the workset display.

Worksets are like boxes in which divide different parts of the project, like for example internal walls, external walls, roof, slabs and also the point clouds' survey; they give the possibility to the various subjects to work each on a local model tied to a central model that is updated constantly through the synchronization of files.

Before to start with the modeling step it was essential to organize all data available to us using them properly and smoothly, arriving to the development of the 3D model of the existing condition. We have not immediately started to model, but we have organized the building information model according to the discipline, that is according to the sector in which we went to work (Architectural, Structural, System, etc.) and according to the different phases of work of the refurbishment of the former thermal power plant. So, the Project Browser has been modified to view the information in the preferred way.

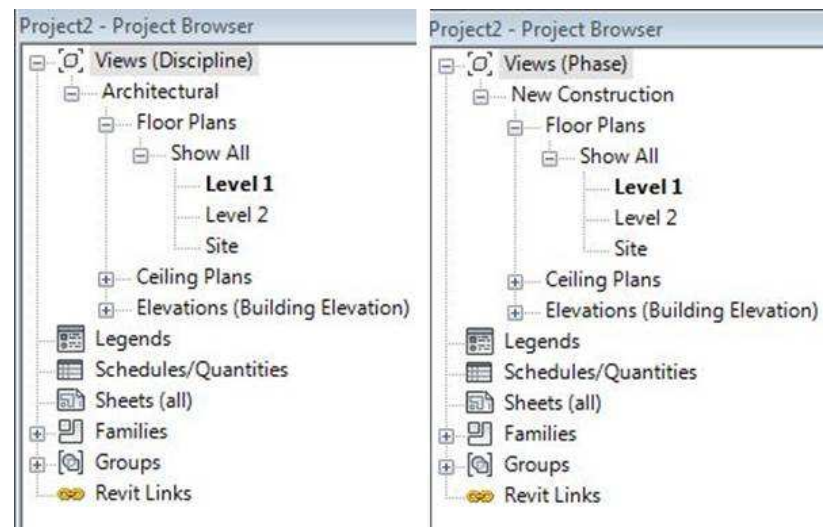

Figure 7. Browser organization of the 3D model.

Also we have assigned to each element the temporal phase related to the time when the element compare in the structure, and for this reason the database can be used for the construction phase of the project (BIM 4D). For this study we dedicated the first phase to the point clouds' survey and after that we created the existing phase, associating each element to the correct phase.

With such a large amount of data, the work organization and the data management have become very important: for this reason the parametric model was organized in the appropriate way, considering each discipline and each phase in the construction process.

In this way once filled data in the information model, professionals can visualize it and can use it for specific 
calculation, avoiding errors, reducing time costs, obtaining good results.

As said before, the first step was to check the consistency between laser scanner survey and archival documents. Therefore, we created the structural grids and, fortunately, from its overlap with the point cloud exists a good fidelity. The grids were created according to the type of element and the type of source that has allowed its localization.

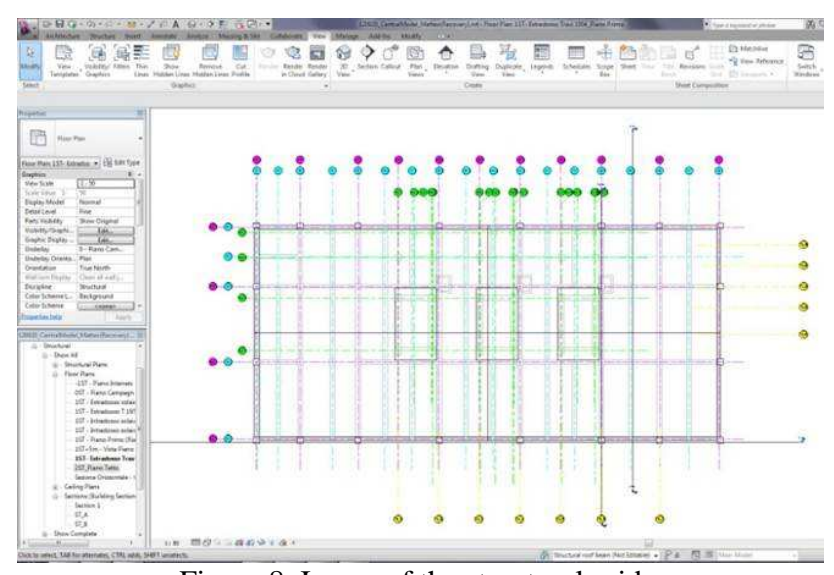

Figure 8. Image of the structural grids.

Since the model was populated with data from different source, some design parameters were created: these are useful to the designer because in this way he can assign to each element the source from which it is derived.

Although there are obvious advantages arising from the point clouds for the development of the architectural model, however there are also some disadvantages such as the need for use of PC able to manage large quantities of data in short time and the not error free interoperable process. The presence of the point clouds, the topographic survey and the design documents was crucial for the development of the parametric model because has greatly facilitated the data processing and their inclusion in the graphic database. The data management according to the BIM process will require the creation of a reference template to be used for the refurbishment of the existing buildings.

\section{RESULTS}

With this study it is clear how the BIM can give a great contribute to transform an existing building in a smart building and InnovANCE project is an opportunity for the AEC Industry to change the way of conceive the construction process.

During the first year of research the first results obtained is undoubtedly the organization among professionals to develop a shared model with Revit. In order to organize the work in a better way considering all the construction process identifying subjects which have to share information to better deliver the BIM process.

Analyzing the former thermal plant of Politecnico di Torino gave the possibility to test the BIM process on existing building, using data coming from different type of survey and archival documents. In this way we discovered how it is more difficult manage a large quantity of data to develop a parametric model related an existing building than a new building.

However, this study will allow us to develop this theme also to refurbish the cultural building heritage.

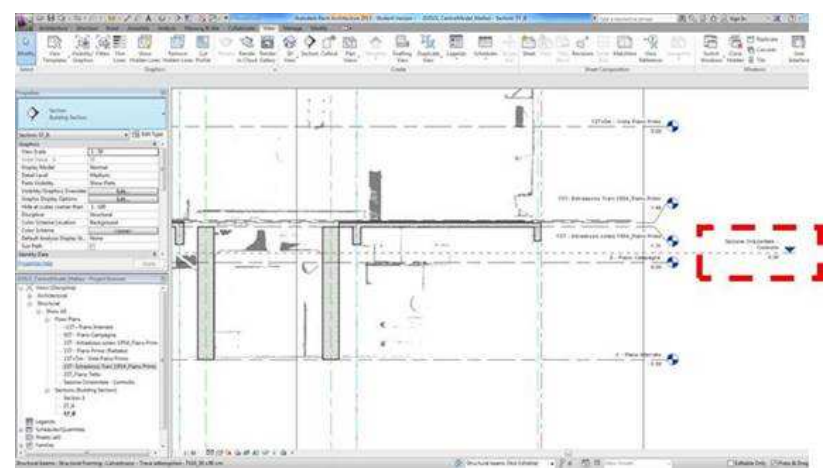

Figure 8. Image of the control level.

Analyzing the step of the point cloud management, we have inserted a level of control to correctly identify the spaces and the elements. We choose to use a level as a functional horizontal section to facilitate the display of elements that are visible from point cloud as previously anticipated. The implementation of these control levels has proved very useful because some components were difficult to detect and would be complex insert the object in the right space.

At present we have created the model of the existing building starting from survey data. We know how the organization of the information is important in a BIM process; this is also a goal of InnovANCE that aims to standardize alphanumeric codes that are able to identify building components and building materials. So by the end of the project, to each BIM object will be associated to an InnovANCE code able to identify one way the building component within the project, becoming part of the InnovANCE database. This will be possible with the Add-in which is in a beta phase in Autodesk Revit.

\section{CONCLUSIONS}

The need to create a database of free access containing all the information useful to the AEC Industry should be considered as the first step in the renewal of this sector in Italy.

The current traditional system fails to meet the increasingly demanding needs of performance capabilities in terms of energy reduction.

In the InnovANCE archive, for each phase of the process, will be encoded uniquely named and described all of procedures and products in the AEC Industry through systems for collecting shared information to all professionals. In this way will be possible to optimize the energy performance of the building and also the building process. Certainly, at present we spent a lot of time to analyze the method which allow to transform an existing building in a smart building and also we have tested the first step of a refurbishment; in the next year will be possible focus on the other phases. More tests are needed to test the 3D model in specific applications, deepening the interoperability issue, arriving to define guidelines for the correct use of BIM for the refurbishment of the existing buildings. As introduced before, existing buildings have a number of major difficulties than new buildings, but the use of new technologies mentioned above gives the opportunity for professionals to manage data, querying the 3Dmodel through a iterative and interactive steps that characterize the BIM process.

The elapsed time was essential to set the job properly and to achieve the objective of the project for subsequent research. Additional time is necessary to apply an iterative methodology essential for new and existing building, specially to translate the results into a first draft of standard BIM guideline valid for the Italian market. 


\section{REFERENCES}

References from Books:

Osello A., Dov'è più diffuso del BIM?, in Osello A., The Future of Drawing with BIM for Engineer and Architects. Flaccovio, Palermo, 2012, pp. 67-101.

References from Other Literature:

Adan A., Xiong X, Akinci B., Huber B, May, 2013 Automatic creation of semantically rich $3 D$ building models from laser scanner data, in Automation in Construction, Vol. 31, ,pp. 325337.

Brusaporci S, Centofanti M., Continenza R, Trizio I.,2012, Sistemi Informativi Architettonici per la gestione, tutela e fruizione dell'edilizia storica. Atti 16a National Conference ASITA - Fiera di Vicenza 6-9 novembre 2012.

Brilakisa, I., Lourakisb, M., Sacksc, R., Silvio Savaresed, S., Christodouloue, S., Teizera, J., Atefe Makhmalbafa, A. 2010. Toward automated generation of parametric BIMs based on hybrid video and laser scanning data. Elsevier, Advanced Engineering Informatics, Vol 24, Issue 4, pp 456-465.

\section{References from websites}

"SMART 2020: Enabling the low carbon economy in the information age",2008 The climate group, http://www.smart2020.org/publications/, (28 June 2013)

Autodesk Wikihelp http://wikihelp.autodesk.com/Revit/enu/2012/Help/Revit_User's Guide/2214-Collabor2214/2269-Working 2269/2274Managing2274 (28 June 2013)

National BIM Library http://www.nationalbimlibrary.com/ website (28 June 2013) 